\title{
Statistical study of thermoradiative and photovoltaic cells based on a two-level model
}

\author{
J. J. Fernández ${ }^{1}$ (iD
}

Received: 26 June 2021 / Accepted: 26 October 2021 / Published online: 18 December 2021

(c) The Author(s) 2021

\begin{abstract}
We use a two-level energy model to understand the conversion process that takes place in thermoradiative cells and to compare it with the conversion process that happens in photovoltaic cells. In this way, we show that in both kinds of converters the conversion process can be studied as the succession of a change in the populations of the levels that occur at constant chemical potential and a change in the value of the chemical potential of the two levels that happens while keeping their populations constant. As an application of the model, we will discuss why in thermoradiative cells the open-circuit voltage is negative while it is positive in photovoltaic cells. We also show that the expression for the open-circuit voltage is the same in both kinds of cells but that due to the values of the temperatures it is negative in thermoradiative cells and positive in photovoltaic ones.
\end{abstract}

Keywords Photovoltaic energy conversion $\cdot$ Thermoradiative energy conversion $\cdot$ Statistical physics $\cdot$ Energy device

Mathematics Subject Classification 05.70.Ln · 05.20.-y

\section{Introduction}

Thermoradiative cells (TRCs) and photovoltaic cells (PVCs) $[1,2]$ are two different devices that generate power using the radiative energy transfer between reservoirs that are kept at constant temperatures. Both TRCs and PVCs are made using semiconductors, and they work in a very similar way. The main difference in their working mode is that while TRCs work at high temperature and exchange photons with a very low temperature environment, PVCs stay at room temperature and receive photons from a very hot source (the Sun).

TRCs are, somehow, a much more new concept than PVCs. Very recently, Strandberg analyzed the performance of far-field TRCs [3] and Samthanam and Fan [4] proved experimentally their working principles. In this last work, it was found that TRCs work with an efficiency much lower than the one predicted in Strandberg's work. In order to improve the performance of TRCs, some authors [5-8]

\footnotetext{
J. J. Fernández

jjfernandez@fisfun.uned.es

1 Dep. Física Fundamental, Universidad Nacional de Educación a Distancia, Paseo de la Senda del Rey, 28040 Madrid, Spain
}

considered the possibility of placing a heat sink between the TRC and its environment. This idea derived from the concept of thermophotovoltaic devices showed that it is possible to improve the performance of TRCs. In fact in thermophotovoltaic cells, using the impedance matching condition [7] and by means of a common design for the PVC absorbed and the hot emitter that emits photons toward the PVC, it is possible to maximize the heat transfer between those two parts of the cell and, consequently, to maximize the cell output power [6-10]. The same can be done in TRCs where it is necessary to have a common design of the cell emitter and the cold sink that receives the photons emitter from the hot cell $[6,8]$.

In this work we study some fundamental issues of thermoradiative energy conversion. To do it, we present a simple description of the thermoradiative energy conversion using simple reasonings of statistical physics. Simple models in physics are very interesting to understand some of the fundamental properties of physical systems and to achieve knowledge about them. Following this thinking, we believe that it is always of interest to have easy-to-follow articles that allow to understand the principles of physical processes. In the past years, this way of thinking was applied for the understanding of the photovoltaic energy conversion [11-15] with 
a great success and we think that it is interesting to apply it to the understanding of new energy conversion techniques such as the thermoradiative energy conversion.

In order to properly present the characteristics of thermoradiative energy conversion, we will simultaneously consider those of photovoltaic conversion. In this way, the reader of this work will be able to get a very clear idea of how these two types of energy conversion are linked by a common fundamental physical description. Thus, thermoradiative and photovoltaic energy conversion can be understood as counterparts of each other as it has already been claimed by some authors [16-18]. Moreover, our exposition clarifies the role that the Carnot factor has, in the description of the thermoradiative energy conversion, a topic that has not been treated in deep in the literature.

According to some previous works, a simple picture having two steps

- Creation of an excitation by light absorption.

- Charge separation and a subsequent splitting of Fermi levels,

is enough to understand some of the fundamentals of photovoltaic conversion. Equally a simple picture of thermoradiative conversion can be achieved assuming the two steps,

- Electron de-excitation and photon emission.

- Charge separation and a subsequent splitting of Fermi levels.

In both cases, and to simplify the reading of the article, we will assume that the excitations/recombinations occur between electrons and holes that exist in the conduction and valence bands of a semiconductor.

It is important to note that both thermoradiative and photovoltaic conversions are affected by other physical processes besides those mentioned. Thus, TRCs do not only emit photons but also absorb them as the temperature of their surroundings is different to $0 \mathrm{~K}$. Similarly, photovoltaic cells (PVCs) are not at $0 \mathrm{~K}$ so they emit photons toward their surroundings while working. In this work, for the sake of simplicity, we will consider that these two processes (absorptions in TRCs and emissions in PVCs) involve a very small amount of photons and that their existence does not affect considerably the statistical distribution of electrons in the semiconductor.

As said, both TRCs and PVCs work interacting to an external field of photons with a temperature $T_{\mathrm{p}}$ that is different to the cell temperature $T_{\mathrm{s}}$. In TRCs $T_{\mathrm{p}}<T_{c}$ what increases in them the number of recombinations to a value that is greater than that of the equilibrium situation. Consequently, in TRCs the lower (higher) energy levels have bigger (smaller) populations than in equilibrium. In PVCs the situation is the opposite. The cell now interacts with photons with a temperature $T_{\mathrm{p}}>T_{\mathrm{s}}$. This interaction increases the number of excitations in the system, so the population of the energy levels of high (low) energy increases over (drops below) the value of equilibrium.

Once we are in the situations described in the preceeding paragraph, electronic currents are created in both TRCs and PVCs. In TRCs hot electrons are injected in the conduction band thus creating an overpopulation. As this situation happens having the cell connected to a cooler environment, the cell tries to cool down. If we force the only way to lose energy in the TRC is through radiative transitions, we will get many electrons to de-excite radiatively to the valence band. Once the electrons are in the valence band, charge separation occurs. In TRCs this process happens as the electrons that have a big temperature diffuse toward the contact that exist in the valence band. Consequently, an electrical current circulates in the TRC that has opposite direction to that of the current of PVCs. This process corresponds to the separation of charges, which, as we see in TRC cells, is somewhat different from how it is in PVC cells. This is so because in PVCs electrons are separated in the built-in electric field of the junction that drives the electrons and holes to contacts of opposite sign (electrons are driven to the cell $n$-side and holes to the p-side), thus creating the electronic current.

As it was said in the preceeding paragraph, in both cases exist electron separation in the pn-junction of the cell. A simple picture of this is achieved considering that once separated both electrons and holes are driven by the internal forces to different particle reservoirs. These two particle reservoirs can be considered to be at the same temperature but at different chemical potential [19]. It is that separation of the chemical potential what gives the cell output voltage. As we will see later, a fundamental difference between TRCs and PVCs is that the chemical potential difference is negative in the first ones and positive in the second ones.

Our work is organized as follows. In Sect. 2 we present a simple, but general, model that is used to understand the conversion in both TRCs and PVCs as the two step processes mentioned above. Our presentation intends to be didactic so we will try to highlight at all times the differences between the physical processes that occur in TRCs and PVCs. In Sect. 3 we present some conclusions about the models presented for TRCs and PVCs in this work and about how they compare.

\section{Statistical model for thermoradiative energy conversion}

Our simple model for both TRCs and PVCs consists of a two-electronic-level system with energies $E_{1}$ and $E_{2}$ with $E_{1}>E_{2}$ that according to Fig. 1 exchange photons in 


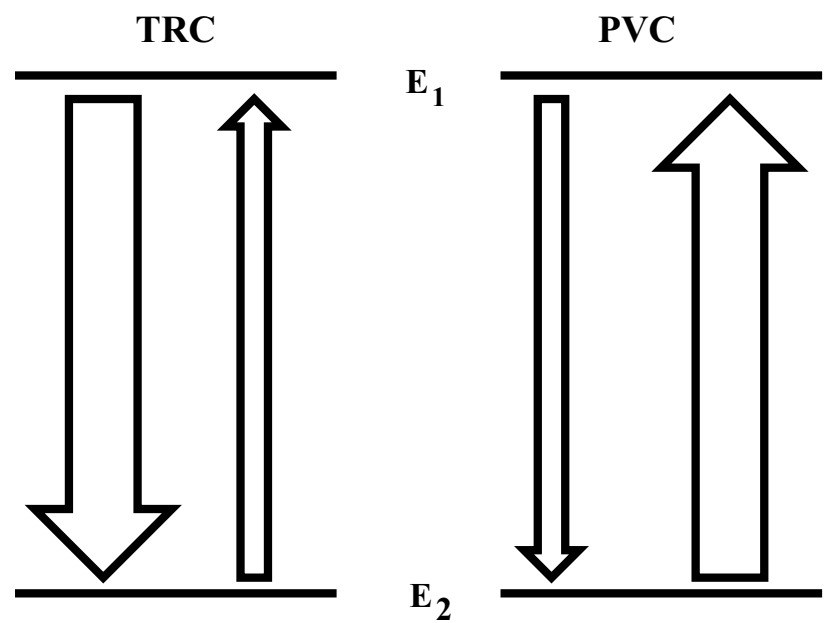

Fig. 1 Schematic diagram of the electronic system used to model TRCs and PVCs. In TRCs the number of recombinations is much bigger than the one of excitations. In PVCs we find the opposite situation; now the number of electronic excitations is much bigger than the number of recombinations

radiative processes. We assume that initially this system is in thermal equilibrium at the same temperature $T_{\mathrm{s}}$ of its surroundings so there is no net exchange of photons between the cell and the surroundings. In this situation the population of the levels $E_{1}$ (population of electrons) and $E_{2}$ (population of holes) are

$p_{i}=\frac{1}{1+s_{i}} \quad$ with $\quad s_{i}=\exp \left[\frac{E_{i}-\mu}{T_{\mathrm{s}}}\right]$.

Here $i=1,2$ denote the two levels of the system or electrons (1) and holes (2); $\mu$ is the electrochemical potential. As we are in an equilibrium situation, $\mu$ is the same for electrons and holes. The two probabilities $p_{1}$ and $p_{2}$ satisfy $p_{1}+p_{2}=1$, so we know that $p_{2}=1-p_{1}$. This equality can be used to write down $s_{1} \times s_{2}=1$ what leads to

$E_{1}+E_{2}=2 \mu \rightarrow \mu=\frac{E_{1}+E_{2}}{2}$.

Equation (2) shows that in equilibrium the chemical potential of the system is in the middle of the energy bandgap that separates the $E_{1}$ and $E_{2}$ levels. This equality that was deduced before for PVCs [19] is also valid for TRCs.

Let us now drive the cells out of equilibrium letting them to interact to their surroundings. For TRCs the interaction to the environment causes the de-excitation of photons from $E_{1}$ to $E_{2}$ and the emission of photons to the surroundings of the cell. PVCs cells are driven out of the equilibrium assuming that they absorb photons coming from a hot source such as the sun. This interaction causes that a part of the electrons that are in the level $E_{2}$ are excited to the level $E_{1}$.

In both cells the interaction to the external field causes a change in $p_{1}$ and $p_{2}$; that now take the values $p_{1}^{*}$ and $p_{2}^{*}$. Proceeding in analogy to the equilibrium situation, we assume

$p_{i}^{*}=\frac{1}{1+s_{i}^{*}} \quad$ with $\quad s_{i}^{*}=\exp \left[\frac{E_{i}-\mu_{i}^{*}}{T_{\mathrm{p}}}\right]$.

Let us note that the two populations $p_{1}^{*}$ and $p_{2}^{*}$ are still given by the Fermi-Dirac distribution function but that now the distribution is characterized by the temperature $T_{\mathrm{p}}$ of the photon field interacting with the cell. It is important to stress that for TRCs $T_{\mathrm{p}}<T_{\mathrm{s}}$ and for PVCs $T_{\mathrm{p}}>T_{\mathrm{s}}$. Although we know that $p_{1}^{*} \neq p_{1}$ and $p_{2}^{*} \neq p_{2}$, the two probabilities $p_{1}^{*}$ and $p_{2}^{*}$ still satisfy $p_{1}^{*}+p_{2}^{*}=1$ what results in

$s_{1}^{*} \times s_{2}^{*}=1 \quad$ or $\quad E_{1}+E_{2}=\mu_{1}^{*}+\mu_{2}^{*}$.

Considering that the interaction of the cell with the radiation of the photon field occurs long enough for the system to reach thermal equilibrium, we can assume that $\mu_{1}^{*}=\mu_{2}^{*}$ and therefore $\mu_{1}^{*}=\mu_{2}^{*}=\mu$. This means that the Fermi level that characterizes the system does not change when it interacts with external radiation. This result is true for both PVCs and TRCs.

However, the effect of connecting the two kind of cells to the external radiation is not the same. On the one hand, in TRCs the external field of photons is characterized by a temperature $T_{\mathrm{p}}<T_{\mathrm{s}}$ (let us think about the example given by Strandberg for the operation of TRCs where they exchange photons to the sky [3]) so the interaction leads to generate de-excitations in the cell. This reduces the population of the energy level $E_{1}$ while increases that of the energy level $E_{2}$. On the other hand, in PVCs that are connected to a hot photonic field, the temperature of the external radiation field is $T_{\mathrm{p}} \approx T_{\text {Sun }}=5870 \mathrm{~K}$. As $T_{\mathrm{p}}>T_{\mathrm{s}}$ the interaction to the photon increases $p_{1}$ and reduces $p_{2}$, i.e., it induces excitations in the PVC.

To model the formation of electronic currents in both TRCs and PVCs, we will assume that each of the two levels of the system $E_{1}$ and $E_{2}$ is connected to a different particle reservoir. We will also consider that the two reservoirs are characterized by having the same temperature and that it coincides with $T_{\mathrm{p}}$, the temperature of the photon field that interacts with the cell. Furthermore, we will assume that the two reservoirs are characterized by different chemical potentials. Thus, the chemical potentials are $\mu_{1}^{\prime}$ for the reservoir connected to the energy level $E_{1}$ and $\mu_{2}^{\prime}$ for the one connected to the energy level $E_{2}$. We also now consider that the formation of the electric current in the cell (it does not matter if it is thermoradiative or photovoltaic) occurs in such a way that the probabilities of occupation of the levels $E_{1}$ and $E_{2}$ are preserved. That is, during the functioning of the 
cell, the populations $p_{1}^{\prime}$ and $p_{2}^{\prime}$ of the two levels are those that were determined by the interaction between the cell and the external field of photons. However, and as is common in works on solar cells, we will calculate $p_{1}^{\prime}$ and $p_{2}^{\prime}$ considering that the temperature characterizing the Fermi-Dirac distribution is $T_{\mathrm{s}}$. If this is done, we must also consider that the chemical potentials $\mu_{1}^{\prime}$ and $\mu_{2}^{\prime}$ are different from $\mu_{1}$ and $\mu_{2}$ and that in general $\mu_{1}^{\prime} \neq \mu_{2}^{\prime}$. Doing this we also know that $\mu_{1}^{\prime}\left(\mu_{2}^{\prime}\right)$ is the unique chemical potential at the $\mathrm{n}$-side (p-side) of the junction [19].

To calculate the values of the two chemical potentials $\mu_{1}^{\prime}$ and $\mu_{2}^{\prime}$, we use the condition of having constant populations in the levels $E_{1}$ and $E_{2}$ Thus, the conditions $p_{1}^{\prime}=p_{1}^{*}$ and $p_{2}^{\prime}=p_{2}^{*}$ stand. From them we obtain,

$\mu_{1}^{\prime}=\frac{T_{\mathrm{s}}}{T_{\mathrm{p}}} \mu+E_{1}\left(1-\frac{T_{\mathrm{s}}}{T_{\mathrm{p}}}\right)$

and

$\mu_{2}^{\prime}=\frac{T_{\mathrm{s}}}{T_{\mathrm{p}}} \mu+E_{2}\left(1-\frac{T_{\mathrm{s}}}{T_{\mathrm{p}}}\right)$

These two conditions are the same for TRCs and for PVCs. However, they have different implications since in TRCs $T_{\mathrm{p}}<T_{\mathrm{s}}$ and in PVCs $T_{\mathrm{p}}>T_{\mathrm{s}}$. For PVCs we have $T_{\mathrm{s}} / T_{\mathrm{p}}<1$, so the factor within the brackets appearing in (5) and (6) has the form of the Carnot efficiency of a reversible heat engine working between two heat reservoirs at $T_{\mathrm{p}}$ and $T_{\mathrm{s}}$. However, in TRCs we have $T_{\mathrm{s}} / T_{\mathrm{p}}>1 \mathrm{so}\left(1-\frac{T_{\mathrm{s}}}{T_{\mathrm{p}}}\right)<0$ is not a Carnot factor.

Doing now the difference $\mu_{1}^{\prime}-\mu_{2}^{\prime}$, we obtain,

$q V_{\text {voc }}=\mu_{1}^{\prime}-\mu_{2}^{\prime}=\left(E_{1}-E_{2}\right)\left[1-\frac{T_{\mathrm{s}}}{T_{\mathrm{p}}}\right]$

This equation shows that the cell open-circuit voltage $V_{\text {voc }}=\left(\mu_{1}^{\prime}-\mu_{2}^{\prime}\right) / q$ is smaller in TRCs than in PVCs. Moreover, in TRCs we have $V_{\text {voc }}<0$.

Finally, we present in Fig. 2 two thermodynamic diagrams of the populations of the energy levels $E_{1}$ and $E_{2}$ in TRCs (a) and PVCs (b) in terms of the chemical potential $\mu$. In the two panels of the figure, we represent in black the population of the level $E_{1}$ and in red that of the level $E_{2}$. We use continuous lines for the populations when the system is in equilibrium and dashed ones when it is in contact to the external radiation. According to Fig. 2, the conversion happening in both TRCs and PVCs can be understood as the combination of an isochemical process (process marked as $a$ in the two panels of the figure) where the population of the level of bigger energy $E_{1}$ reduces in TRCs and increases in PVCs while the chemical potential is kept constant. We also mark process $b$ (that corresponds to the change separation) in which the populations
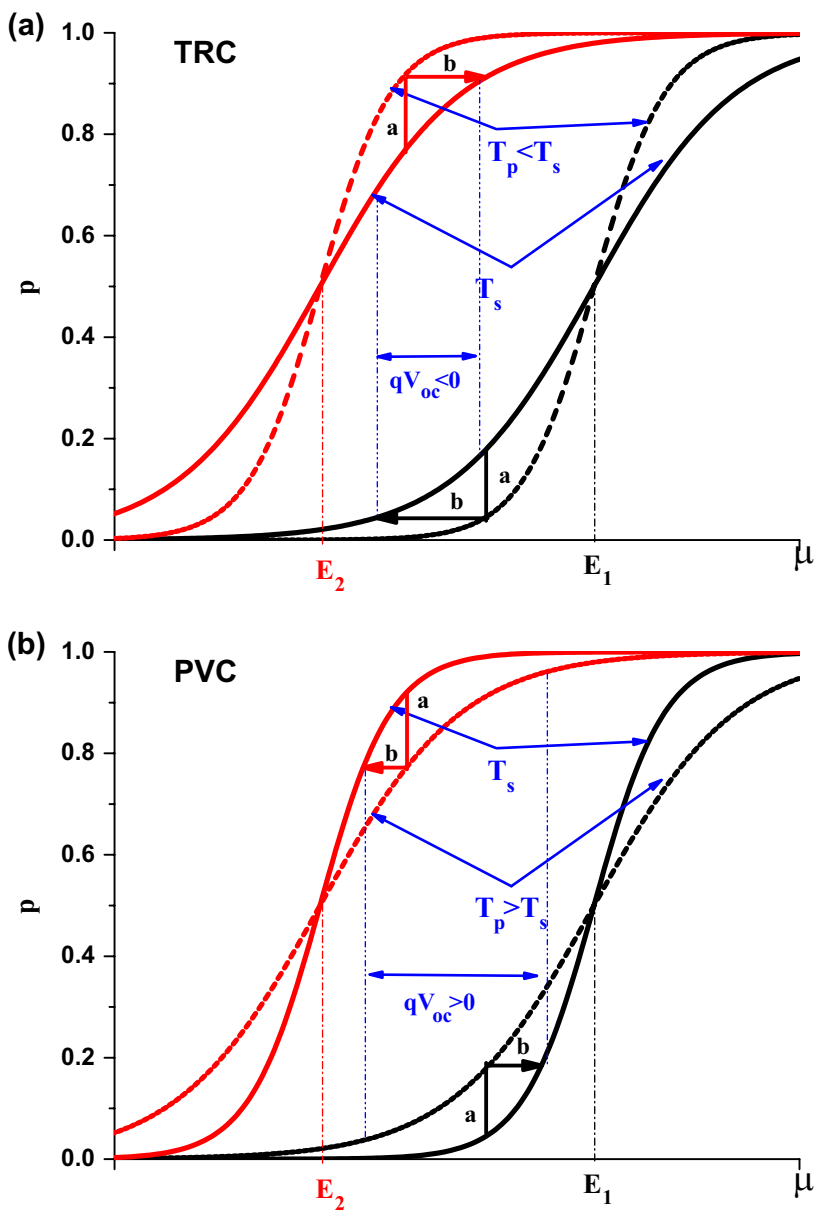

Fig. 2 Thermodynamic $p$ versus $\mu$ diagram of the energy conversion happening in TRCs (a) and PVCs (b). In both panels the solid lines correspond to the (equilibrium) electronic populations $p_{1}$ and $p_{2}$ at the temperature $T_{\mathrm{s}}$. In (a) the dashed line represents the electronic populations $p_{1}^{*}$ and $p_{2}^{*}$ at $T_{\mathrm{p}}<T_{\mathrm{s}}$. In (b) the dashed line represents the electronic populations $p_{1}^{*}$ and $p_{2}^{*}$ at $T_{\mathrm{p}}>T_{\mathrm{s}}$

of the two energy levels remains constant while their chemical potentials change. In TRCs the chemical potential $\mu_{1}^{\prime}$ of the level $E_{1}$ is at a lower energy (it moves to the left in the figure) than it was in the system at the initial equilibrium temperature $T_{\mathrm{s}}$. At the same time, in TRCs the chemical potential $\mu_{2}^{\prime}$ associated to $E_{2}$ also changes; it moves toward bigger energies (it moves to the right in Fig. 2). Thus, in the final (working situation) of TRCs we have $\mu_{2}^{\prime}>\mu_{1}^{\prime}$ and $q V_{\text {voc }}<0$. This behavior is opposite to the one found in PVCs where $\mu_{1}^{\prime}$ moves toward higher energies, $\mu_{2}^{\prime}$ toward lower ones and $q V_{\mathrm{voc}}>0$.

\section{Conclusions}

In this work we used a simple statistical model of two energy levels to understand some basic features of thermoradiative energy conversion. In order to properly establish the context 
of our presentation and to demonstrate that the operation of thermoradiative cells is similar to that of photovoltaic cells, we have presented our discussion comparing the processes that occur in the two kind of cells. Our analysis has led us to see the similarities and differences that exist between photovoltaic and thermoradiative cells.

Thus, we have understood that in thermoradiative cells, as in photovoltaic ones, the energy conversion can be viewed as a two-step process. In the first step the populations of the two levels change. The change happens keeping the chemical potential of the two levels constant. In thermoradiative cells this step represents the part of the conversion where electrons de-excite from the upper energy levels to the lower energy ones. The process entails an increase of the population of the levels of lower energy and a reduction of the population of those of higher energy. In photovoltaic cells the first step represents the process where electrons excite from the lower energy levels to the higher ones. Thus, the population of the lower energy levels decreases and that of the bigger energy increases. As we see, the interaction to the external field causes opposite effects on the populations of the energy levels of TRCs and PVCs.

In the process of charge separation, and in both kinds of cells, the chemical potentials associated to the high and low energy levels change. In thermoradiative cells the chemical potential of the energy levels with the lower energy increases and that of the energy levels with the higher energy decreases. In normal working situations of thermoradiative cells, the chemical potential of the high energy level is smaller than the one of the other levels. Thus, in TRCs it is said that the difference of the chemical potentials is negative and so it is the cell open-circuit voltage. This situation is the opposite to that found in PVCs where the difference between the two chemical potentials is positive and so it is the cell open-circuit voltage.

Acknowledgements The author thanks the UNED for providing its computational facilities to carry out the work and to Prof. J. E. Alvarellos and Drs. David García Aldea, E. M. Fernández and J. Rodríguez-Laguna. The author acknowledges to the Ministerio de Ciencia e Innovación for financial support through the research Project PID-2019-105182GB-I00.

Funding Open Access funding provided thanks to the CRUE-CSIC agreement with Springer Nature.

Data availability The data that support the findings of this study are available from the corresponding author upon reasonable request.

Open Access This article is licensed under a Creative Commons Attribution 4.0 International License, which permits use, sharing, adaptation, distribution and reproduction in any medium or format, as long as you give appropriate credit to the original author(s) and the source, provide a link to the Creative Commons licence, and indicate if changes were made. The images or other third party material in this article are included in the article's Creative Commons licence, unless indicated otherwise in a credit line to the material. If material is not included in the article's Creative Commons licence and your intended use is not permitted by statutory regulation or exceeds the permitted use, you will need to obtain permission directly from the copyright holder. To view a copy of this licence, visit http://creativecommons. org/licenses/by/4.0/.

\section{References}

1. Shockley, W., Queisser, H.J.: Detailed balance limit of efficiency of pn junction solar cells. J. Appl. Phys. 32, 510-519 (1961)

2. Würfel, P.: Physics of Solar Cells. Wiley-VCH, Weinheim (2005)

3. Strandberg, R.: Theoretical efficiency limits for thermoradiative energy conversion. J. Appl. Phys. 117, 055105 (2015)

4. Santhanam, P., Fan, S.: Thermal-to-electrical energy conversion by diodes under negative illumination. Phys. Rev. B 93, 161410 (2016)

5. Hsu, W.-C., Tong, J.K., Liao, B., Huang, Y., Boriskina, S.V., Chen, G.: Entropic and near-field improvements of thermoradiative cells. Sci. Rep. 6, 34837 (2016)

6. Lin, C., Wang, B., Teo, K.H., Zhang, Z.M.: Near-field enhancement of thermoradiative devices. J. Appl. Phys. 122, 143102 (2017)

7. Lin, C., Wang, B., Teo, K.H., Bandaru, P.: Application of impedance matching for enhanced transmitted power in a thermophotovoltaic system. Phys. Rev. Appl. 7, 034003 (2017)

8. Wang, B., Lin, C., Teo, K.H., Zhang, Z.M.: Thermoradiative device enhanced by near-field coupled structures. J. Quantum Spectrosc. Radiat. Transf. 196, 10 (2017)

9. Karalis, A., Joannopoulos, J.D.: Squeezing near-field thermal emission for ultra-efficient high-power thermophotovoltaic conversion. Sci. Rep. 6, 141108 (2016)

10. Karalis, A., Joannopoulos, J.D.: Temporal coupled-mode theory model for resonant near-field thermophotovoltaics. Appl. Phys. Lett. 107, 141108 (2015)

11. de Vos, A.: Thermodynamics of Endoreversible Solar Energy Conversion. Oxford University Press, Oxford (1992)

12. Fahrenbruch, A., Bube, R.: Fundamentals of Solar Cells. Academic Press, New York (1983)

13. Jordan, A.G., Lade, R.W., Scharfetter, D.L.: Am. J. Phys. 31, 490 (1963)

14. Landsberg, P.T.: Solid-State Electron. 18, 1043 (1975)

15. Baruch, P., Parrott, J.E.: J. Phys. D. 23, 739 (1990)

16. Buddhiraju, S., Santhanam, P., Fan, S.: Proc. Natl. Acad. Sci. U.S.A. 115(16), E3609 (2017)

17. Fernández, J.J.: J. Appl. Phys. 128, 044501 (2020)

18. Fernández, J.J.: IEEE J. Photovolt. 11(2), 437-443 (2021)

19. Landsberg, P.T., Markvart, T.: Solid State Electron. 42, 657 (1998)

Publisher's Note Springer Nature remains neutral with regard to jurisdictional claims in published maps and institutional affiliations. 